\title{
Multi-agent neoadjuvant chemotherapy improves response and survival in patients with resectable pancreatic cancer
}

\author{
Paige Blinn ${ }^{1}$, Ravi Shridhar ${ }^{2}$, Taylor Maramara ${ }^{1}$, Jamie Huston ${ }^{3}$, Kenneth Meredith ${ }^{1,3}$ \\ ${ }^{1}$ Florida State University College of Medicine, Tallahassee, FL, USA; ${ }^{2}$ Florida Hospital Cancer Institute, Orlando, FL, USA; ${ }^{3}$ Sarasota Memorial \\ Institute for Cancer Care, Sarasota, FL, USA \\ Contributions: (I) Conception and design: P Blinn, R Shridhar, T Maramara, K Meredith; (II) Administrative support: None; (III) Provision of study \\ materials or patients: None; (IV) Collection and assembly of data: J Huston; (V) Data analysis and interpretation: P Blinn, R Shridhar, T Maramara, \\ K Meredith; (VI) Manuscript writing: All authors; (VII) Final approval of manuscript: All authors. \\ Correspondence to: Kenneth Meredith, MD, FACS. Professor, Florida State University College of Medicine, Tallahassee, FL, USA; Director, \\ Gastrointestinal Oncology, Sarasota Memorial Institute for Cancer Care, 1950 Arlington Street, Suite 101, Sarasota, FL 34239, USA. Email: \\ Dr.Kenneth-Meredith@smh.com; kensurg@hotmail.com.
}

Background: We sought to examine the impact of neoadjuvant chemotherapy (NCT), single agent (SA) or multi-agent (MA) chemotherapy, and chemoradiation (NCRT) on response and survival in pancreatic cancer. Methods: Utilizing the National Cancer Database, we identified patients who underwent resection of the pancreatic head for adenocarcinoma [2006-2013]. Overall survival (OS) analysis was performed using the Kaplan-Meier method. Multivariable cox proportional hazard models (MVA) and propensity score matching (PSM) were developed to identify predictors of survival. For upfront surgery (UFS), OS was limited to receipt of adjuvant treatment.

Results: We identified 26,563 patients who underwent pancreatic head resection: UFS =23,877, NCRT $=1,482$, and NCT $=1,204$. MA-NCT was utilized in 77\% and after PSM, 52\%. There was improved R0 resections and 30-day mortality associated with neoadjuvant therapy compared to UFS. Overall response rate to neoadjuvant therapy was $24 \%$. The highest response rate seen with MA-NCRT. Response rates for SANCT, MA-NCT, SA-NCRT, and MA-NCRT were $11.5 \%, 18.1 \%, 27.5 \%$, and $33.1 \%(\mathrm{P}=0.01)$. However, OS was improved with neoadjuvant therapy regardless of response compared to UFS ( $\mathrm{P}=0.03)$. After PSM, the median OS for UFS, SA-NCT, MA-NCT, SA-NCRT, and MA-NCRT was 21.9, 21.5, 29.8, 25.3, and 25.8 months in all patients $(\mathrm{P}=0.001)$. MVA after PSM demonstrated that only MA-NCT was associated with decreased mortality while increasing age, higher Charlson-Deyo index, N1, higher grade, tumor size, and positive margins were associated with higher mortality.

Conclusions: There was improved OS associated with MA-NCT in pancreatic cancer patients compared to UFS with adjuvant therapy. OS was improved regardless of response to therapy.

Keywords: Pancreatic cancer; neoadjuvant therapy; National Cancer Database (NCDB); multiagent chemotherapy (MAC)

Submitted Sep 20, 2019. Accepted for publication Nov 22, 2019.

doi: 10.21037/jgo.2019.12.03

View this article at: http://dx.doi.org/10.21037/jgo.2019.12.03

\section{Introduction}

Pancreatic cancer is the fourth leading cause of cancer mortality in the United States, with a 5-year survival rate of $8 \%$ (1). Complete surgical resection remains the only curative treatment for resectable tumors. However, only about $15-20 \%$ of tumors are considered candidates for surgical intervention at the time of diagnosis (2). Current recommendations by the National Comprehensive Cancer Network (NCCN) for resectable pancreatic cancer is to 
perform definitive surgery followed by adjuvant therapy (3).

The use of adjuvant therapy has been shown to be advantageous in its ability to improve overall survival (OS) and decrease tumor recurrence rates following surgical resection (4-8). However, adjuvant therapy has its associated disadvantages, largely attributed to patients who are unable to complete the planned therapy course due to treatment related co-morbidities and local and distant disease progression $(9,10)$. This is an aggressive cancer associated with early micro-metastases. One study found that approximately $38 \%$ of patients who underwent complete pancreatic tumor resection had recurrent disease due to possible micro-metastases not identified preoperatively, leading to significantly shorter OS compared to patients without early recurrence (9.3 vs. 26.3 months) (11).

Though its effectiveness is still being debated, neoadjuvant therapy seeks to combat these shortcomings of the current standard of care. Neoadjuvant therapy has been shown to potentially improve resection rates through tumor down-staging, subsequently increasing the number of eligible candidates for curative surgery. Also due to postoperative complications and chemotherapy associated toxicities, the use of adjuvant therapy may be delayed or even prevented leading to consequential disease progression. The utilization of neoadjuvant therapy will allow more patients to receive all necessary multimodal treatments. Neoadjuvant therapy has also been shown to target occult micro-metastases that can significantly contribute to disease recurrence. Importantly this treatment sequence can also improve patient selection by excluding those that would not benefit from curative resection due to undetected metastatic disease or aggressive disease $(9,10,12)$.

With multiple studies demonstrating improved survival rates with the emerging application of neoadjuvant therapy compared to adjuvant therapy, it becomes increasingly important to determine the ideal treatment sequence and modality $(2,10,13,14)$. There is currently little data to support an optimal treatment regimen when utilizing neoadjuvant chemotherapy (NCT) agents (with or without radiation, $\pm \mathrm{RT}$ ). Our goal is to identify the impact of single agent (SAC) vs. multi-agent (MAC) chemotherapy $\pm \mathrm{RT}$ on the survival of patients with resectable pancreatic cancer.

\section{Methods}

\section{Patients}

This retrospective study was approved and deemed exempt by the Sarasota Memorial Hospital institutional review board (16-ONC-03). The National Cancer Database (NCDB) is a database that collects patient data from $>1,500$ commission-accredited cancer programs centers in the United States. More than 70 percent of this data represents newly diagnosed cancer cases nationwide. We queried the NCDB for patients with a diagnosis of adenocarcinoma of the pancreatic head who underwent upfront surgery (UFS) as well as those who received NCT $\pm \mathrm{RT}(\mathrm{NT}+\mathrm{RT}$ and NT - RT respectively) followed by surgery.

\section{Statistics}

Baseline univariate comparisons of patient characteristics between the UFS, NT + RT, and NT - RT patients were made for continuous variables using both the MannWhitney $U$ and Kruskal Wallis tests as appropriate. To compare categorical variables a Pearson's Chi-square test was used when appropriate. OS was defined as the time of diagnosis to death or last contact. Survival time was censored for patients alive at the end of the study period. Survival outcomes were analyzed using Kaplan-Meier method which generated OS curves and estimated median survival with $95 \%$ confidence intervals for each group. The log-rank test was used to compare survival distributions across groups.

Multivariable Cox proportional hazard models (MVA) were developed comparing treatment methods [definitive surgery, SAC NCT, SAC neoadjuvant chemoradiation (NCRT), MAC NCT, and MAC NCRT]. Predictors of long-term survival included in the models were age, sex, pathologic T-stage, pathologic $\mathrm{N}$-stage, tumor grade, tumor size, lymph nodes harvested, number of lymph of positive lymph nodes, surgical margins, institution volume, adjuvant therapy and use of induction therapy.

Propensity score matching (PSM) was used to correct for baseline differences among treatment groups to match for age, tumor size, and facility volume. Neoadjuvant therapy $(\mathrm{N}=2,686)$ served as the case group and UFS $(\mathrm{N}=23,877)$ the control group. Matching occurred on a 1:1 basis and only exact matches were allowed. All statistical tests were twosided and $\alpha$ (type I) error $<0.05$ was considered statistically significant. Statistical analysis was performed using SPSS ${ }^{\circledR}$ version $24.0\left(\mathrm{IBM}^{\circledR}\right.$, Chicago, IL, USA).

\section{Results}

We identified 26,563 patients from the NCDB who 
underwent resection of the pancreatic head for cancer of which 1,204 (4.5\%) underwent NCT, 1,482 (5.6\%) underwent NCRT and 23,877 (89.9\%) underwent UFS (Table 1). Significant differences were noted for age, Charlson-Deyo index, tumor size, lymph nodes removed, lymph nodes positive, pathologic T- and $\mathrm{N}$-stage, grade, 30and 90-day mortality, surgical margins, facility volume, and adjuvant therapy. MAC was given to $77 \%$ of patients who were not radiated and in $42 \%$ of radiated patients $(\mathrm{P}<0.001)$. In patients receiving neoadjuvant therapy, the complete response rates were $1.7 \%$ for NCT and $3.1 \%$ for NCRT $(\mathrm{P}<0.001)$. We used PSM analysis of neoadjuvant therapy (NCT and NCRT) versus UFS matched by age, tumor length, and facility volume. After PSM, 3,066 patients were identified with significant differences in lymph nodes removed, lymph nodes positive, pathologic $\mathrm{T}$ - and $\mathrm{N}$-stage, 30-day mortality, surgical margins, and adjuvant therapy. No adjuvant therapy was given in $62 \%$ of patients treated with neoadjuvant therapy compared to $28 \%$ for UFS patients $(\mathrm{P}<0.001)$. MAC was given to $31 \%$ of patients who were not radiated and in $16 \%$ of radiated patients.

Table 2 illustrates response rates by treatment group.
Higher response rates were observed in patients who were radiated and received MAC. Highest response rates were observed in multi-agent (MA)-NCRT patients while the lowest response rates were seen in single agent (SA)-NCT patients. Overall response rate to neoadjuvant therapy was $24 \%$. The highest response rate seen with MA-NCRT. Response rates for SA-NCT, MA-NCT, SA-NCRT, and MA-NCRT were $11.5 \%, 18.1 \%, 27.5 \%$, and $33.1 \%$ $(\mathrm{P}=0.01)$. However, OS was improved with neoadjuvant therapy regardless of response (Figure 1, Table 3). Median OS in UFS, NT with complete response, NT with partial response, and NT with no response was 21.9, 29.8, 28.3, and 24.7 months $(\mathrm{P}=0.03)$. On univariate analysis, there was decreased mortality in patients who received neoadjuvant therapy regardless of response compared to UFS $(\mathrm{P}<0.001)$ before PSM. After PSM, there was decreased mortality in patients achieving a partial response $(\mathrm{P}=0.01)$ and a strongly trending worse survival in the non-responders $(\mathrm{P}=0.06)$.

OS in patients who received NCT and NCRT was compared to UFS who received adjuvant therapy. After PSM, the median OS for UFS, SA-NCT, MA-NCT, SANCRT, and MA-NCRT was 21.9, 21.5, 29.8, 25.3, and

Table 1 Patient characteristics

\begin{tabular}{|c|c|c|c|c|c|c|c|}
\hline Variable & \multicolumn{4}{|c|}{ Non-PSM } & \multicolumn{3}{|c|}{ PSM } \\
\hline $\begin{array}{l}\text { Median age (years) } \\
\text { [range] }\end{array}$ & 64 [31-88] & 64 [25-90] & 67 [18-90] & $<0.001$ & 64 [26-90] & 64 [26-90] & 1.00 \\
\hline Male & $620(51.5)$ & 780 (52.6) & $12,304(51.5)$ & & 802 (52.3) & 787 (51.3) & \\
\hline Female & 584 (48.5) & 702 (47.4) & $11,573(48.5)$ & & 731 (47.7) & 746 (48.7) & \\
\hline Charlson-Deyo & & & & 0.008 & & & 0.61 \\
\hline 0 & $852(70.8)$ & $999(67.4)$ & $15,918(66.7)$ & & $1,021(66.6)$ & 1,036 (67.6) & \\
\hline Tumor length & & & & 0.003 & & & 1.00 \\
\hline$<2 \mathrm{~cm}$ & $179(15.8)$ & $210(15.1)$ & $4,140(18.1)$ & & 239 (15.6) & 239 (15.6) & \\
\hline$>2 \mathrm{~cm}$ & 956 (84.2) & 1,180 (84.9) & 18,728 (81.9) & & $1,294(84.4)$ & 1,294 (84.4) & \\
\hline $\begin{array}{l}\text { Median lymph nodes } \\
\text { removed [range] }\end{array}$ & 17 [0-69] & 12 [0-68] & 14 [0-90] & $<0.001$ & 15 [0-69] & 15 [0-63] & 0.001 \\
\hline
\end{tabular}

Table 1 (continued) 
Table 1 (continued)

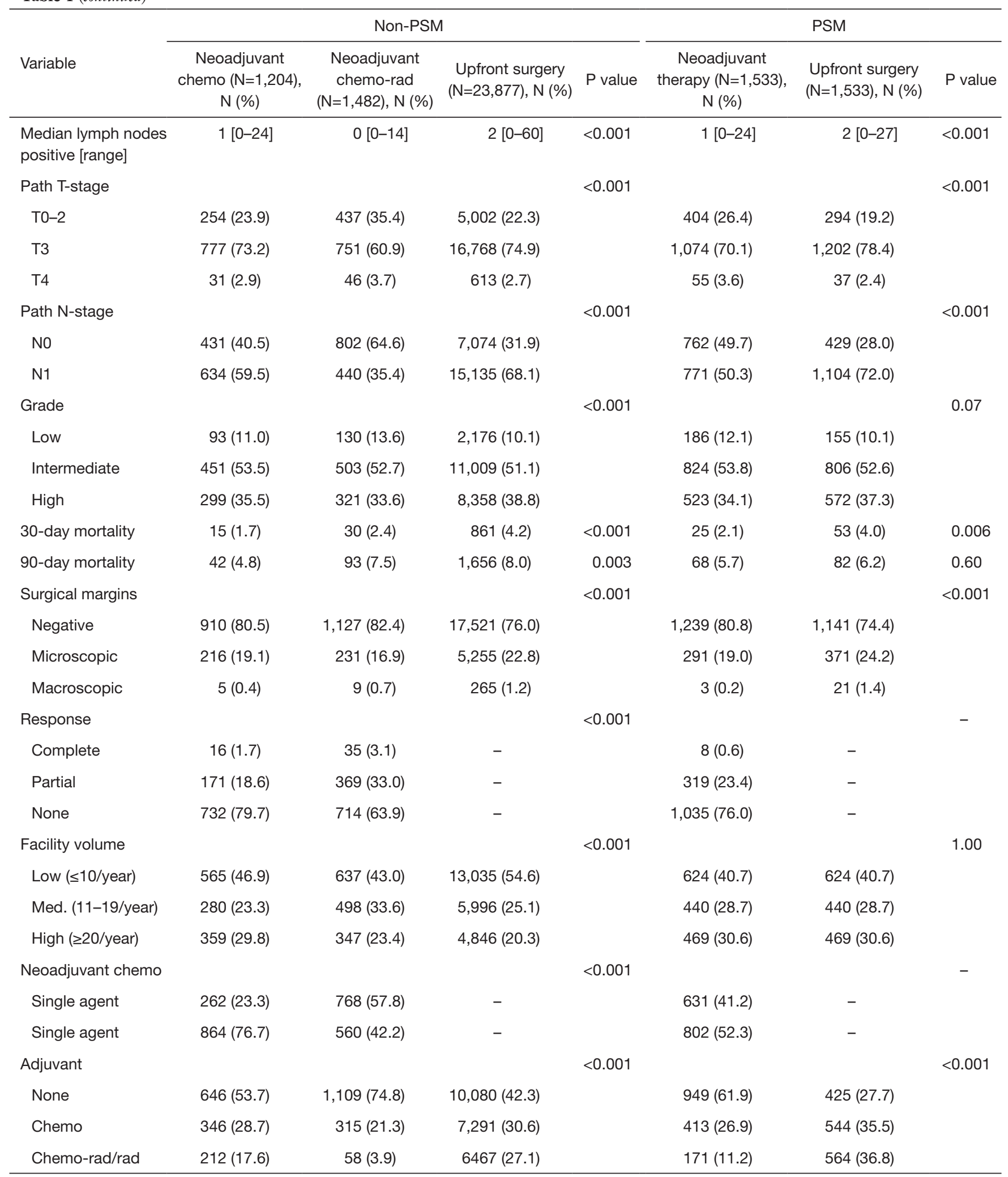

Chemo, chemotherapy; chemo-rad, chemoradiation; rad, radiation; Med., medium. 
Table 2 Pathologic response and neoadjuvant agent

\begin{tabular}{|c|c|c|c|c|c|c|c|c|c|c|c|c|}
\hline \multirow{2}{*}{$\begin{array}{l}\text { Treatment } \\
\text { group }\end{array}$} & \multicolumn{6}{|c|}{ Non-PSM } & \multicolumn{6}{|c|}{ PSM } \\
\hline & $\mathrm{N}(\%)$ & $P$ value & $\mathrm{N}(\%)$ & $P$ value & N (\%) & $P$ value & $\mathrm{N}(\%)$ & $\begin{array}{c}\mathrm{P} \\
\text { value }\end{array}$ & $\mathrm{N}(\%)$ & $P$ value & N (\%) & $P$ value \\
\hline Single agent & & 0.07 & & $<0.001$ & & $<0.001$ & & - & & $<0.001$ & & $<0.001$ \\
\hline SA-NCRT & $17(2.8)$ & & $187(30.7)$ & & 405 (66.5) & & 0 & & $116(27.5)$ & & 306 (72.5) & \\
\hline Multi-agent & & 0.25 & & $<0.001$ & & $<0.001$ & & 0.02 & & $<0.001$ & & $<0.001$ \\
\hline MA-NCT & $15(2.2)$ & & 135 (19.7) & & $536(78.1)$ & & $1(0.2)$ & & $85(18.1)$ & & $384(81.7)$ & \\
\hline MA-NCRT & $15(3.3)$ & & $166(36.5)$ & & 274 (60.2) & & $5(2.0)$ & & $84(33.1)$ & & $165(65.0)$ & \\
\hline MA-NCT & $15(2.2)$ & & 135 (19.7) & & $536(78.1)$ & & $1(0.2)$ & & $85(18.1)$ & & 384 (81.7) & \\
\hline $\begin{array}{l}\text { Neoadjuvant } \\
\text { chemoradiation }\end{array}$ & & 0.63 & & 0.05 & & 0.04 & & 0.01 & & 0.12 & & 0.04 \\
\hline SA-NCRT & $17(2.8)$ & & $187(30.7)$ & & 405 (66.5) & & 0 & & $116(27.5)$ & & $306(72.5)$ & \\
\hline MA-NCRT & 15 (3.3) & & $166(36.5)$ & & $274(60.2)$ & & $5(2.0)$ & & $84(33.1)$ & & $165(65.0)$ & \\
\hline
\end{tabular}

SA, single agent; MA, multi-agent; NCT, neoadjuvant chemotherapy; NCRT, neoadjuvant chemoradiation.

25.8 months in all patients $(\mathrm{P}=0.001)$ (Figure 2). Univariate analysis of the PSM group revealed that increasing age, Charlson-Deyo index, pathologic T- and N-stage, higher grade, tumor size, and positive surgical margins were associated with increased mortality. MA-NCT and MANCRT were associated with decreased mortality, while gender, SA-NCT, SA-NCRT, and facility volumes were not prognostic (Table 3). Multivariate analysis of the PSM group revealed that increasing age, Charlson-Deyo index, pathologic N1, higher grade, tumor size $>2 \mathrm{~cm}$, and positive surgical margins were associated with increased of mortality. MA-NCT was the only factor associated with decreased mortality, while SA-NCT, SA-NCRT, MA-NCRT, gender, pathologic T-stage, and facility volumes were not prognostic (Table 4).

\section{Discussion}

Our data demonstrates an improvement in OS associated with MA-NCT. There was improved R0 resections and 30-day mortality associated with neoadjuvant therapy compared to UFS. Overall response rate to neoadjuvant therapy was $24 \%$. The highest response rate seen with MA-NCRT. However, OS was improved with neoadjuvant therapy regardless of response compared to UFS $(\mathrm{P}=0.03)$. MVA demonstrated that only MA-NCT was associated with decreased mortality while increasing age, higher CharlsonDeyo index, N1, higher grade, tumor size, and positive margins were associated with higher mortality.

The current management recommendation for resectable and borderline resectable pancreatic cancer remains upfront surgical resection followed by adjuvant chemotherapy; radiation may be considered in patients with nodal disease and close or positive margins (3). Gemcitabine has been the standard adjuvant agent for over a decade. PRODIGE, a randomized phase III clinical trial that compared FOLFIRINOX (5-fluorouracil plus leucovorin, irinotecan and oxaliplatin) to gemcitabine in 493 patients with resectable tumors, found that adjuvant therapy with FOLFIRNOX demonstrated an improvement in median OS (54.4 vs. 34.8 months, $\mathrm{P}=0.003$ ). However, FOLFIRNOX was associated higher rates of severe side 


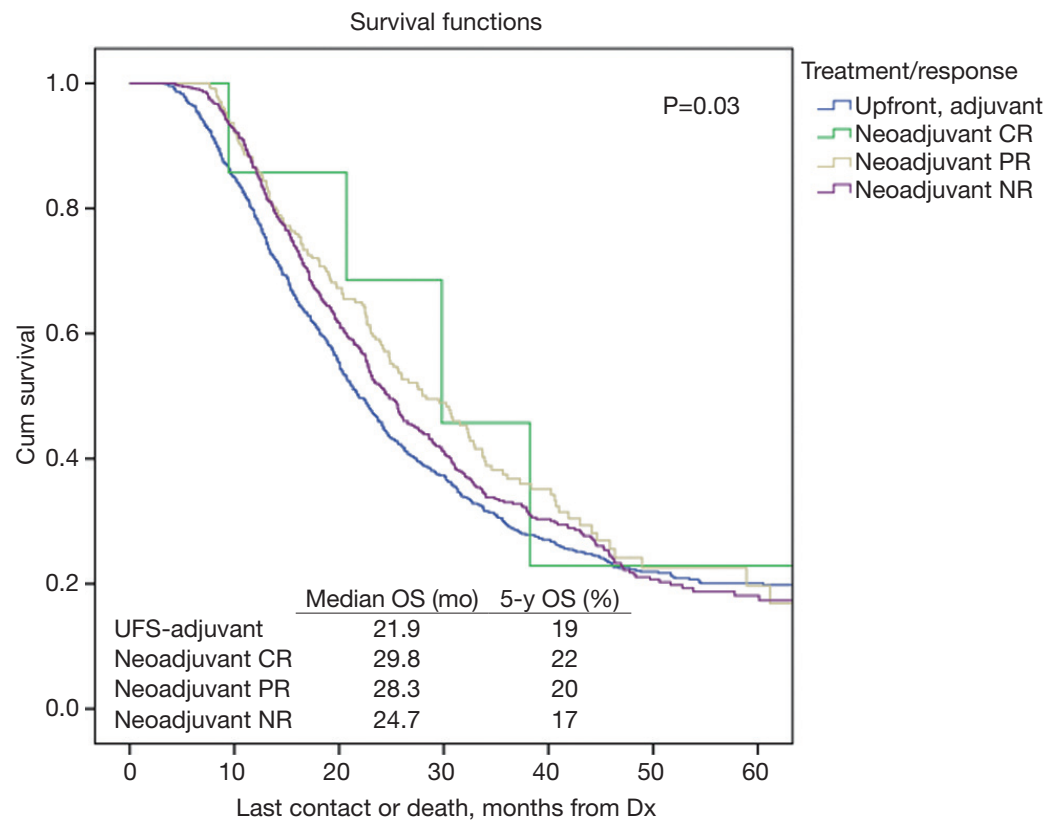

Figure 1 Kaplan-Meier analysis of overall survival by response. UFS, upfront surgery; CR, complete response; PR, partial response; NR, no response; OS, overall survival; Dx, diagnosis.

effects $(76 \%$ vs. $53 \%)(15)$. While this established treatment sequence has shown promising results, it fails a significant proportion of patients who are unable complete all of the necessary adjuvant treatment largely due to postoperative complications and chemo-related toxicities. In the aforementioned PRODIGE study, only $66 \%$ and $79 \%$ of patients received all cycles of adjuvant FOLFIRNOX and gemcitabine treatments, respectively $(\mathrm{P}=0.002)$. This has been corroborated by other authors who support surgical resection followed by adjuvant therapy, though significant selection bias is present due to the exclusion of patients who were not able to receive the entire adjuvant treatment course (up to $60 \%$ in some studies) due to complications (16-19).

Though neoadjuvant therapy remains controversial, it has several theoretical benefits to address the obstacles seen in a surgical resection and adjuvant therapy approach. PREOPANC-1, an ongoing randomized phase III clinical trial with 246 patients, compared preoperative chemoradiation therapy to immediate surgery and has demonstrated that preoperative interventions improved median OS (17.1 vs. 13.7 months, $\mathrm{P}=0.74$ ), delayed disease recurrence (9.9 vs. 7.9 months, $\mathrm{P}=0.023$ ), and had higher two-year survival rates $(42 \%$ vs. $30 \%)$. In patients whose tumor was completely removed, the median OS was significantly higher in the pre-operative chemoradiation group (42.1 vs. 16.2 months) (20). Our data revealed that all four neoadjuvant modalities that we examined ( $\mathrm{SAC} \pm$ $\mathrm{RT}$ and $\mathrm{MAC} \pm \mathrm{RT}$ ) demonstrated an improved median OS rate versus upfront surgical resection followed by adjuvant therapy.

Due to this high percentage of patients not receiving adjuvant treatment, many authors have continued to investigate potential benefits from a neoadjuvant approach. Tzeng et al. compared 167 patients, of which 115 received neoadjuvant therapy and 52 underwent UFS with adjuvant therapy, and found that $83 \%$ of patients who received neoadjuvant therapy were able to complete all multimodal therapy $v s .58 \%$ of patients in the UFS group who were able to complete adjuvant therapy $(\mathrm{P}=0.001)(21)$. Additionally, in the EPSAC-1 and CONKO-001 randomized controlled trials, $50 \%$ and $38 \%$ of patients respectively, either did not start or complete their adjuvant therapy course $(6,22)$. In our data alone, which is a larger series, 10,080 (42\%) patients who underwent UFS did not receive adjuvant therapy $(\mathrm{P}<0.001)$. These results demonstrate the significant number of patients whose adjuvant therapy either is delayed or reduced. This shortcoming can contribute to disease recurrence and decreased median survival $(9,10)$. The use of neoadjuvant therapy helps to maximize the administration 
Table 3 Univariate analysis

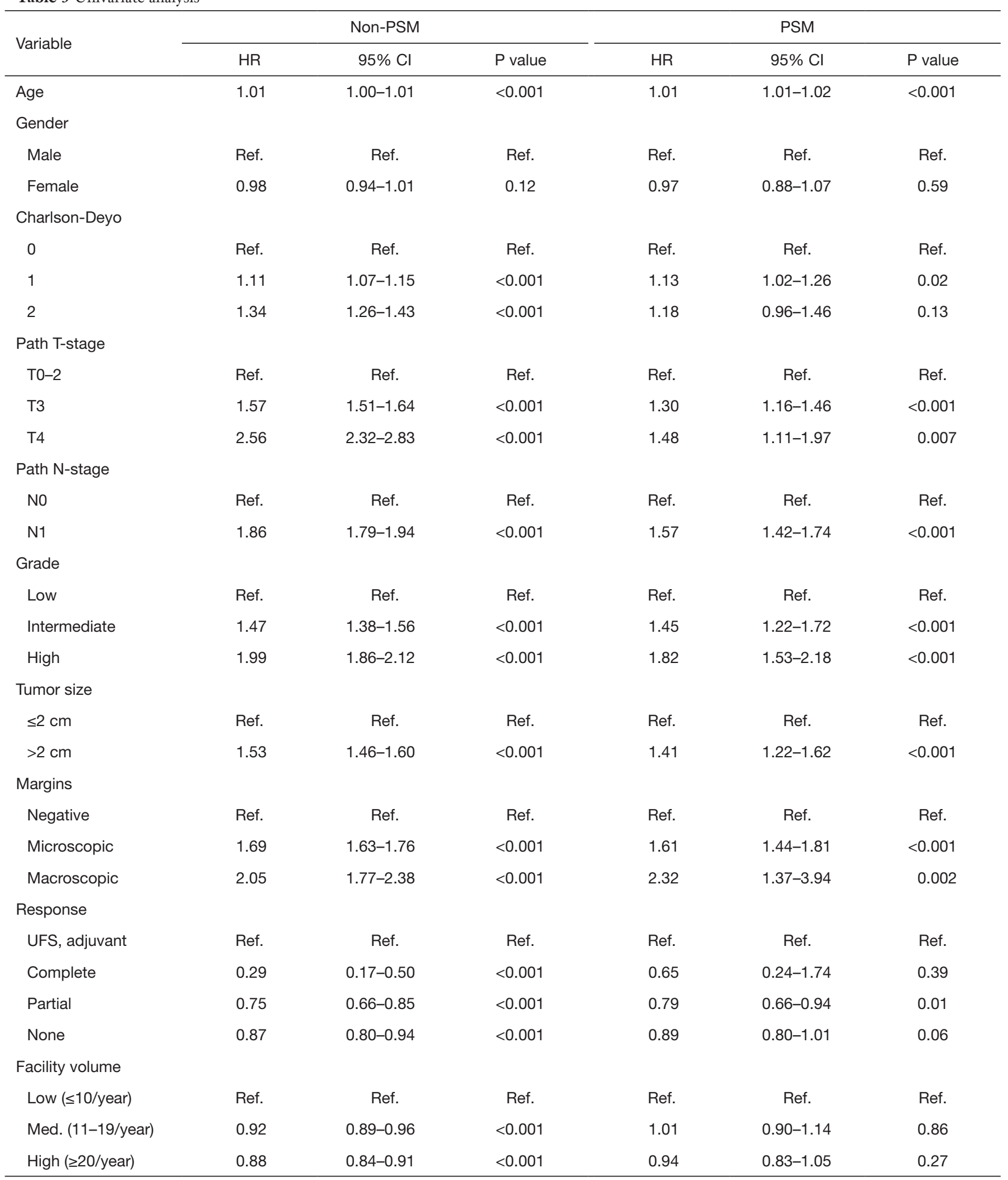

Table 3 (continued) 
Table 3 (continued)

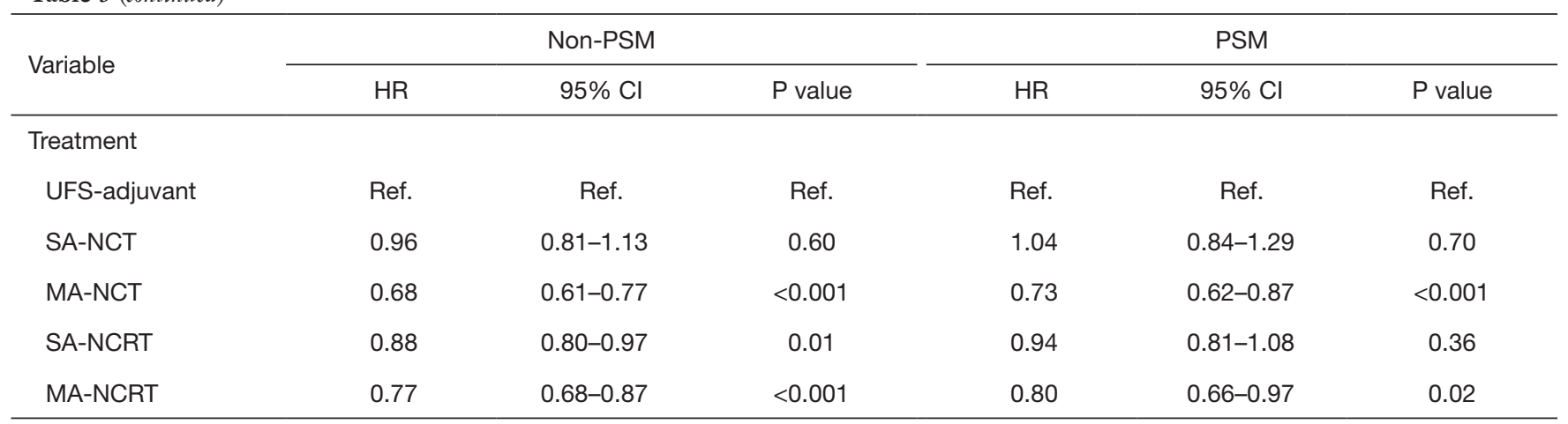

PSM, propensity score matching; HR, hazard ratio; Cl, confidence interval; UFS, upfront surgery; Ref., reference; Med., medium; SA, single agent; MA, multi-agent; NCT, neoadjuvant chemotherapy; NCRT, neoadjuvant chemoradiation.

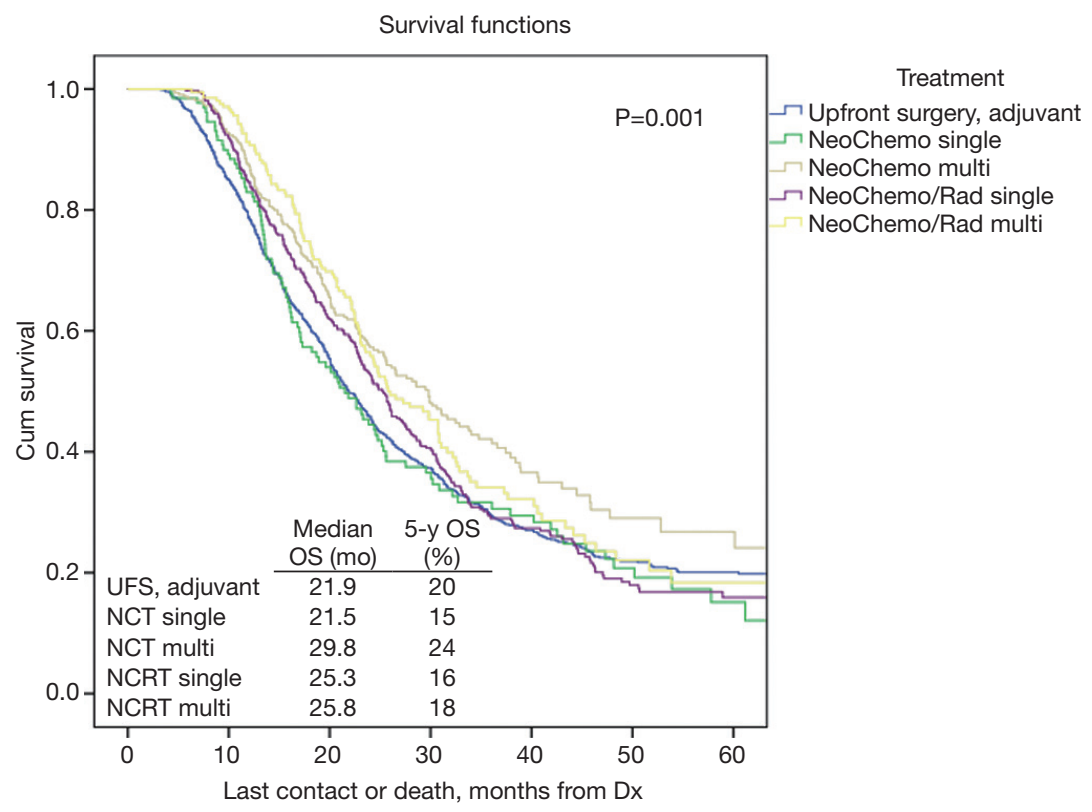

Figure 2 Kaplan-Meier analysis of overall survival by treatment. UFS, upfront surgery; NCT (NeoChemo), single neoadjuvant chemotherapy single agent; NCT (NeoChemo), multi-neoadjuvant chemotherapy multi-agent; NCRT (NeoChemo/Rad), single neoadjuvant chemoradiation single agent; NCRT (NeoChemo/Rad), multi-neoadjuvant chemoradiation multi-agent; Dx, diagnosis.

of systemic chemotherapy to ensure patients receive all necessary components of the multimodal therapy upfront.

Another potential benefit of a neoadjuvant approach is the possibility of downsizing tumors that are considered borderline resectable. A neoadjuvant approach potentially increases the rates of resectability. This remains important given the fact that a limited proportion of patients are considered to have a resectable tumor at the time of diagnosis and surgical resection remains the only curative measure (2). Lim et al. included five prospective neoadjuvant trials that contained borderline resectable pancreatic tumors and found that the surgical resection rates of these tumors ranged from $33-64 \%$ with $\mathrm{R} 0$ sections ranging between $87-$ $100 \%(23-28)$. Though the sample size of these studies were small (ranging from 15-40 patients each), it demonstrates the potential for secondary resectability and achievement of $\mathrm{R} 0$ resection in a greater number of patients.

Negative margins and negative nodal involvement are established predictors of improved survival in patients with pancreatic cancer who underwent curative resection (29-34). 
Table 4 Multivariate analysis

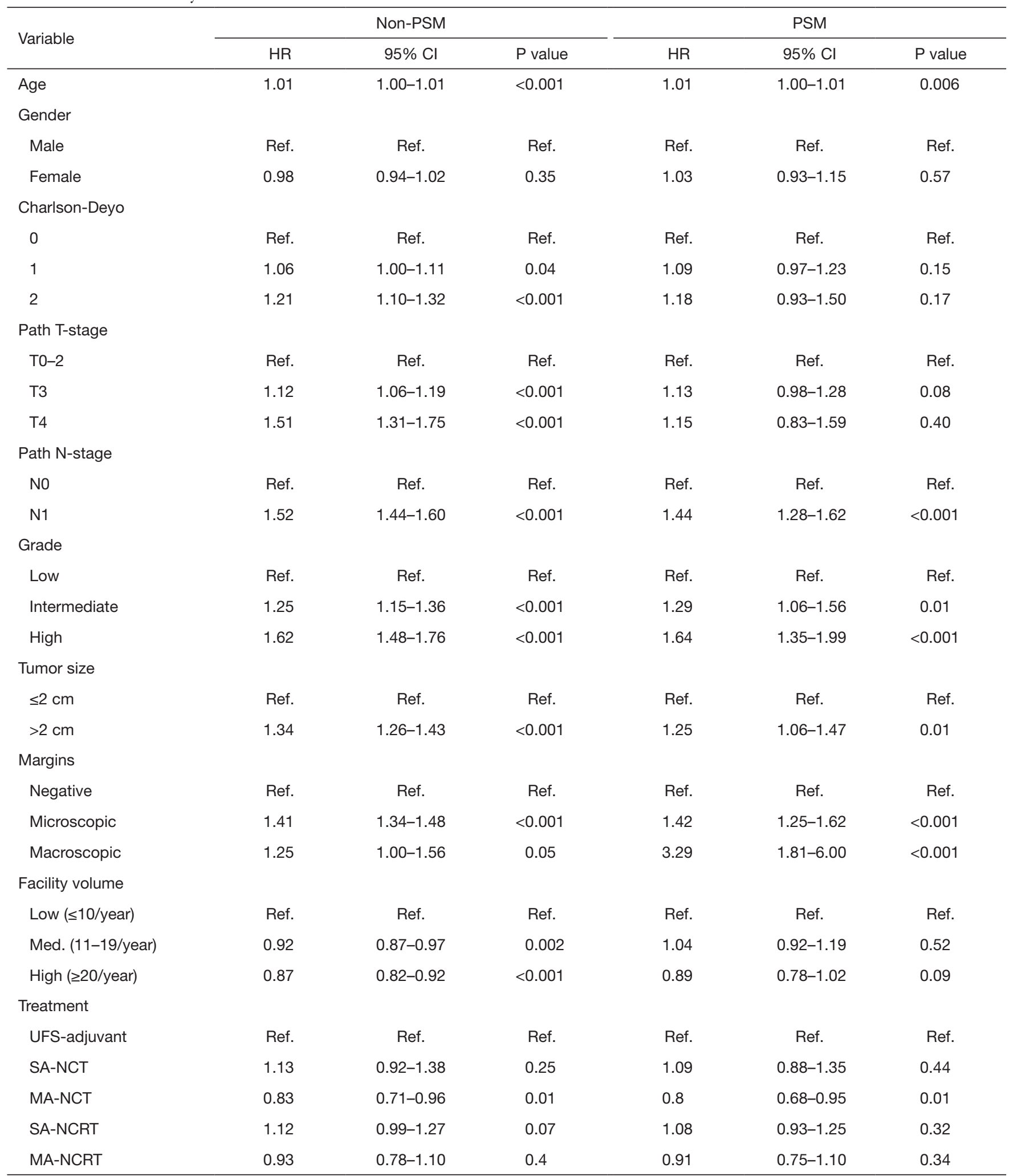

PSM, propensity score matching; HR, hazard ratio; CI, confidence interval; UFS, upfront surgery; Ref., reference; Med., medium; SA, single agent; MA, multi-agent; NCT, neoadjuvant chemotherapy; NCRT, neoadjuvant chemoradiation. 
Our study demonstrated higher R0 resection rates in patients who received neoadjuvant therapy compared to those who underwent UFS (MAC 82.4\%, SAC $80.5 \%$, and surgery $76 \%$ ). We also demonstrated less N1 disease and fewer positive lymph nodes compared to UFS. The presence of micro-metastasis can contribute to locoregional recurrence or distant metastasis leading to poor OS after resection (11). Recognizing this indicates that pancreatic cancer is, in many cases, a systemic disease and therefore necessitates a systemic approach. A potential benefit from a neoadjuvant approach is to allow micro-metastatic disease to declare itself during the course of therapy thereby preventing patients from underdoing a morbid operative without curative benefit.

The optimal neoadjuvant therapy course remains poorly defined. When comparing SA $v s$. multi-agent treatment modalities, we found that MAC with and without radiation had a superior survival benefit. MAC without radiation was also found have the greatest improvement on median survival and 5-year survival across all treatment groups. In a randomized phase III study with 342 patients, Conroy et al. compared FOLFIRINOX to gemcitabine alone in patients with metastatic pancreatic cancer and found that the MAC group had significantly improved median OS vs. monotherapy (11.1 vs. 6.8 months, $\mathrm{P}<0.001$ ) (35). Similarly, in a larger randomized phase III study with 861 patients, Von Hoff et al. concluded increased median OS with nabpaclitaxel plus gemcitabine compared to gemcitabine alone (8.5 vs. 6.7 months, $\mathrm{P}<0.001$ ) (36). We also found significant survival benefit in patients when MAC neoadjuvant approach was adopted.

Our study is clearly limited due to the nature of a retrospective analysis. We attempted to limit the selection bias by including PSM. Our study is also limited in the ability to standardize data input across institutions and monitoring the guidelines used for data collection in the NCDB. Additionally, while we recognized that MAC prolonged median OS, our data does not include which specific chemotherapy regimens were utilized in treatment of our patient population as this data is not included in the NCDB.

\section{Conclusions}

With the relatively low survival rate, even in patients who undergo curative surgical resection, it becomes important to determine the ideal timing and modality of chemotherapeutic and radiation treatment regimens for patients with resectable pancreatic cancer. Adjuvant therapy significantly improved survival for those who complete all of the therapy at the recommended doses. However, those who cannot complete adjuvant therapy due to toxicities or post-operative complications have significant hindrance in their survival. Neoadjuvant therapy potentially addresses these shortcomings. This data supports the use of neoadjuvant MAC without radiation to provide the greatest improvement in the median OS of patients with resectable pancreatic tumors. Ongoing clinical trials will further corroborate our findings and clarify the management of pancreatic cancer.

\section{Acknowledgments}

Funding: None.

\section{Footnote}

Conflicts of Interest: The authors have no conflicts of interest to declare.

Ethical Statement: The authors are accountable for all aspects of the work in ensuring that questions related to the accuracy or integrity of any part of the work are appropriately investigated and resolved. This study was reviewed by the Sarasota Memorial Hospital Institutional Review Board (16-ONC-03) and determined exempt because it does not meet the definition of human subject research.

Open Access Statement: This is an Open Access article distributed in accordance with the Creative Commons Attribution-NonCommercial-NoDerivs 4.0 International License (CC BY-NC-ND 4.0), which permits the noncommercial replication and distribution of the article with the strict proviso that no changes or edits are made and the original work is properly cited (including links to both the formal publication through the relevant DOI and the license). See: https://creativecommons.org/licenses/by-nc-nd/4.0/.

\section{References}

1. Siegel RL, Miller KD, Jemal A. Cancer statistics, 2018. CA Cancer J Clin 2018;68:7-30.

2. Hackert T. Surgery for Pancreatic Cancer after neoadjuvant treatment. Ann Gastroenterol Surg 2018;2:413-8. 
3. Tempero MA, Malafa MP, Al-Hawary M, et al. Pancreatic Adenocarcinoma, Version 2.2017, NCCN Clinical Practice Guidelines in Oncology. J Natl Compr Canc Netw 2017;15:1028-61.

4. Kalser MH, Ellenberg SS. Pancreatic cancer. Adjuvant combined radiation and chemotherapy following curative resection. Arch Surg 1985;120:899-903.

5. Neoptolemos JP, Stocken DD, Bassi C, et al. Adjuvant chemotherapy with fluorouracil plus folinic acid vs gemcitabine following pancreatic cancer resection: a randomized controlled trial. JAMA 2010;304:1073-81.

6. Neoptolemos JP, Stocken DD, Friess H, et al. A randomized trial of chemoradiotherapy and chemotherapy after resection of pancreatic cancer. $\mathrm{N}$ Engl J Med 2004;350:1200-10.

7. Oettle H, Neuhaus P, Hochhaus A, et al. Adjuvant chemotherapy with gemcitabine and long-term outcomes among patients with resected pancreatic cancer: the CONKO-001 randomized trial. JAMA 2013;310:1473-81.

8. Regine WF, Winter KA, Abrams RA, et al. Fluorouracil vs gemcitabine chemotherapy before and after fluorouracilbased chemoradiation following resection of pancreatic adenocarcinoma: a randomized controlled trial. JAMA 2008;299:1019-26.

9. Artinyan A, Anaya DA, McKenzie S, et al. Neoadjuvant therapy is associated with improved survival in resectable pancreatic adenocarcinoma. Cancer 2011;117:2044-9.

10. Heinrich S, Lang H. Neoadjuvant Therapy of Pancreatic Cancer: Definitions and Benefits. Int J Mol Sci 2017. doi: 10.3390/ijms18081622.

11. Fischer R, Breidert $M$, Keck T, et al. Early recurrence of pancreatic cancer after resection and during adjuvant chemotherapy. Saudi J Gastroenterol 2012;18:118-21.

12. Puleo F, Marechal R, Demetter P, et al. New challenges in perioperative management of pancreatic cancer. World J Gastroenterol 2015;21:2281-93.

13. Mokdad AA, Minter RM, Zhu H, et al. Neoadjuvant Therapy Followed by Resection Versus Upfront Resection for Resectable Pancreatic Cancer: A Propensity Score Matched Analysis. J Clin Oncol 2017;35:515-22.

14. Takahashi C, Shridhar R, Huston J, et al. Correlation of tumor size and survival in pancreatic cancer. J Gastrointest Oncol 2018;9:910-21.

15. Conroy T, Hammel P, Hebbar M, et al. Unicancer GI PRODIGE 24/CCTG PA.6 trial: A multicenter international randomized phase III trial of adjuvant mFOLFIRINOX versus gemcitabine (gem) in patients with resected pancreatic ductal adenocarcinomas. J
Clin Oncol 2018. doi: 10.1200/JCO.2018.36.18_suppl. LBA4001.

16. Aloia TA, Aloia TE, Lee JE, et al. Delayed recovery after pancreaticoduodenectomy: a major factor impairing the delivery of adjuvant therapy? J Am Coll Surg 2007;204:347-55.

17. Corsini MM, Miller RC, Haddock MG, et al. Adjuvant radiotherapy and chemotherapy for pancreatic carcinoma: the Mayo Clinic experience (1975-2005). J Clin Oncol 2008;26:3511-6.

18. Herman JM, Swartz MJ, Hsu CC, et al. Analysis of fluorouracil-based adjuvant chemotherapy and radiation after pancreaticoduodenectomy for ductal adenocarcinoma of the pancreas: results of a large, prospectively collected database at the Johns Hopkins Hospital. J Clin Oncol 2008;26:3503-10.

19. Winter JM, Brennan MF, Tang LH, et al. Survival after resection of pancreatic adenocarcinoma: results from a single institution over three decades. Ann Surg Oncol 2012;19:169-75.

20. Van Tienhoven G, Versteijne E, Suker M, et al. Preoperative chemoradiotherapy versus immediate surgery for resectable and borderline resectable pancreatic cancer (PREOPANC-1): A randomized, controlled, multicenter phase III trial. J Clin Oncol 2018. doi: 10.1200/ JCO.2018.36.18_suppl.LBA4002.

21. Tzeng CW, Tran Cao HS, Lee JE, et al. Treatment sequencing for resectable pancreatic cancer: influence of early metastases and surgical complications on multimodality therapy completion and survival. J Gastrointest Surg 2014;18:16-24; discussion 24-5.

22. Oettle H, Post S, Neuhaus P, et al. Adjuvant chemotherapy with gemcitabine vs observation in patients undergoing curative-intent resection of pancreatic cancer: a randomized controlled trial. JAMA 2007;297:267-77.

23. Lim KH, Chung E, Khan A, et al. Neoadjuvant therapy of pancreatic cancer: the emerging paradigm? Oncologist 2012;17:192-200.

24. Massucco P, Capussotti L, Magnino A, et al. Pancreatic resections after chemoradiotherapy for locally advanced ductal adenocarcinoma: analysis of perioperative outcome and survival. Ann Surg Oncol 2006;13:1201-8.

25. Mehta VK, Poen JC, Ford JM, et al. Protracted venous infusion 5 -fluorouracil with concomitant radiotherapy compared with bolus 5 -fluorouracil for unresectable pancreatic cancer. Am J Clin Oncol 2001;24:155-9.

26. Patel M, Hoffe S, Malafa M, et al. Neoadjuvant GTX chemotherapy and IMRT-based chemoradiation for 
borderline resectable pancreatic cancer. J Surg Oncol 2011;104:155-61.

27. Small W Jr, Berlin J, Freedman GM, et al. Full-dose gemcitabine with concurrent radiation therapy in patients with nonmetastatic pancreatic cancer: a multicenter phase II trial. J Clin Oncol 2008;26:942-7.

28. Stokes JB, Nolan NJ, Stelow EB, et al. Preoperative capecitabine and concurrent radiation for borderline resectable pancreatic cancer. Ann Surg Oncol 2011;18:619-27.

29. Howard TJ, Krug JE, Yu J, et al. A margin-negative R0 resection accomplished with minimal postoperative complications is the surgeon's contribution to longterm survival in pancreatic cancer. J Gastrointest Surg 2006;10:1338-45; discussion 1345-6.

30. Lim JE, Chien MW, Earle CC. Prognostic factors following curative resection for pancreatic adenocarcinoma: a population-based, linked database analysis of 396 patients. Ann Surg 2003;237:74-85.

31. Morales-Oyarvide V, Rubinson DA, Dunne RF, et al.

Cite this article as: Blinn P, Shridhar R, Maramara T, Huston J, Meredith K. Multi-agent neoadjuvant chemotherapy improves response and survival in patients with resectable pancreatic cancer. J Gastrointest Oncol 2020;11(5):1078-1089. doi: 10.21037/jgo.2019.12.03
Lymph node metastases in resected pancreatic ductal adenocarcinoma: predictors of disease recurrence and survival. Br J Cancer 2017;117:1874-82.

32. Raut CP, Tseng JF, Sun CC, et al. Impact of resection status on pattern of failure and survival after pancreaticoduodenectomy for pancreatic adenocarcinoma. Ann Surg 2007;246:52-60.

33. Richter A, Niedergethmann M, Sturm JW, et al. Longterm results of partial pancreaticoduodenectomy for ductal adenocarcinoma of the pancreatic head: 25-year experience. World J Surg 2003;27:324-9.

34. Tarantino I, Warschkow R, Hackert T, et al. Staging of pancreatic cancer based on the number of positive lymph nodes. Br J Surg 2017;104:608-18.

35. Conroy T, Desseigne F, Ychou M, et al. FOLFIRINOX versus Gemcitabine for Metastatic Pancreatic Cancer. New England Journal of Medicine 2011;364:1817-25.

36. Von Hoff DD, Ervin T, Arena FP, et al. Increased survival in pancreatic cancer with nab-paclitaxel plus gemcitabine. N Engl J Med 2013;369:1691-703. 